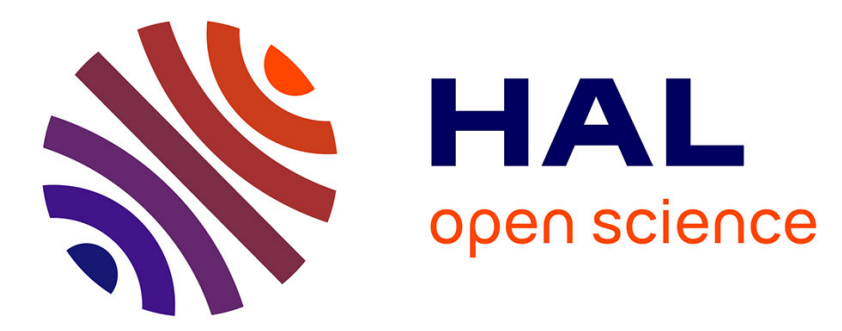

\title{
Probabilistic design and uncertainty quantification of the structure of a monopile offshore wind turbine
}

\author{
Abraham Nispel, Stephen Ekwaro-Osire, João Paulo Dias, Americo Cunha Jr
}

\section{To cite this version:}

Abraham Nispel, Stephen Ekwaro-Osire, João Paulo Dias, Americo Cunha Jr. Probabilistic design and uncertainty quantification of the structure of a monopile offshore wind turbine. ASME 2019 International Mechanical Engineering Congress and Exposition (IMECE2019), Nov 2019, Salt Lake City, United States. hal-02388512

\section{HAL Id: hal-02388512 https://hal.science/hal-02388512}

Submitted on 2 Dec 2019

HAL is a multi-disciplinary open access archive for the deposit and dissemination of scientific research documents, whether they are published or not. The documents may come from teaching and research institutions in France or abroad, or from public or private research centers.
L'archive ouverte pluridisciplinaire HAL, est destinée au dépôt et à la diffusion de documents scientifiques de niveau recherche, publiés ou non, émanant des établissements d'enseignement et de recherche français ou étrangers, des laboratoires publics ou privés. 


\title{
PROBABILISTIC DESIGN AND UNCERTAINTY QUANTIFICATION OF THE STRUCTURE OF A MONOPILE OFFSHORE WIND TURBINE
}

\author{
Abraham Nispel, Stephen Ekwaro-Osire1, João Paulo Dias \\ Department of Mechanical Engineering \\ Texas Tech University \\ Lubbock, TX, USA
}

\author{
Americo Cunha Jr. \\ Institute of Mathematics and Statistics \\ Rio de Janeiro State University \\ Rio de Janeiro, RJ, Brazil
}

\begin{abstract}
Despite the increasing demand for offshore energy, structural components of offshore wind turbines (OWT), such as the tower and foundation, are considered the most critical parts of the turbine. In fact, uncertainties regarding load conditions, soil and structural properties highly undermine the OWT structural reliability. In this scenario, in order to obtain more accurate results, rigorous probabilistic analyses are necessary. In this study, a probabilistic analysis of the dynamic response of a monopile OWT is conducted by using a systematic uncertainty quantification (UQ) framework to deal with the uncertainty assessment of the model input parameters. The proposed dynamic model computes the dynamic response of the turbine due to wind and waves loads on the monopile structure utilizing a simple cantilever beam analytical model. The distributions of the model input parameters are determined using (1) nonparametric statistics for a large dataset, and (2) the maximum entropy principle for a small dataset. Monte Carlo simulations are performed to propagate the uncertainties of the model inputs and to determine the system reliability expressed in terms of their probability of failure for the serviceability limit state design criterion. Finally, to demonstrate the shortcomings of traditional approaches that assume standard distributions to model uncertainties, a UQ approach modeling the uncertainties of the parameters using normal distributions is contrasted with our framework. From the results, significant differences between the distribution shape and values of the probability of failure can be observed; thus, it demonstrates the importance of developing probabilistic frameworks with systematic $U Q$ to have more realistic approximations of the reliability of the OWT structure.
\end{abstract}

Keywords: offshore wind turbines, probabilistic analysis, uncertainty quantification, structural reliability.

\footnotetext{
${ }^{1}$ Corresponding author: stephen.ekwaro-osire@ttu.edu
}

\section{INTRODUCTION}

Renewable energies have arisen as a solid alternative to satisfy the growing demand for energy around the globe. Factors such as global warming, economic volatility of fossil fuels and technology developments have made renewable energies competitive to conventional energies. Among the variety of clean sources of energy, offshore wind power is the sector that has grown significantly over the last decades [1]. In contrast with onshore applications, offshore wind energy present significant advantages, such as the lower risk for human life, fewer space constraints, less turbulent winds, and wind availability at higher speeds [2]. Despite the associated benefits of offshore wind energy, the overall cost of the projects is higher than for the onshore counterparts. Offshore wind turbines (OWT) deal with issues regarding extreme environmental loads, and complicated geographical locations, which result in a noticeable impact over the design, installation, and maintenance of offshore wind plants [3]. In terms of design, the turbine foundation is critical and may reach a cost of the order of 25 to $35 \%$ of the whole system [4], [5]. Foundations are the elements of the structure that connect the upper part of the structure to the ground. Their function is to transmit the combinations of loads exerted over the structure to the ground and to provide the sufficient stiffness to reduce deformations at the lower level, which in turn, prevent large deformations at the highest parts of the structure. Nevertheless, the installation of OWT towers in poor quality terrains requires deeper substructure penetrations into the seabed, as well as stiffer and taller substructures to meet the design standards. Hence, despite the considerable the research made on the design of OWT foundations, there are no significant improvements in the system reliability, which leads to the necessity of research in this area.

As seen in the literature, a significant number of studies which are primarily focused on the behavior of monopilefoundations are found in comparison with other substructures. In 
fact, monopiles are the most utilized OWT foundation type in shallow waters, (i.e., less than 30 meters depth) due to their simplicity in terms of manufacturing, installation, and deinstallation [2], [6]. The majority of the current models of monopile foundations rely on studies developed essentially for monopiles substructures designed for either oil or gas offshore platforms [7], [8]. However, these approaches are questioned by other authors because the intended purpose for an oil platform differs significantly from an OWT. Because of that, it is still necessary to identify the most sensitive parameters for OWT foundation that have a major impact on the foundation reliability.

The standards to design foundations included in the main design codes are primarily divided into four criteria, namely, ultimate-limit state (ULS), resonance or the fundamental frequency criterion, fatigue limit state (FLS), and serviceability limit state (SLS) [4]-[6]. Among these criteria, the SLS, which define the maximum deflections and rotations that the structure may have, has been described as the most stringent. The SLS criteria gain more attention particularly due to the tendency of the offshore wind industry to build taller towers and larger blades which leads to excessive deflection at the wind turbine hub [3], [4], [6]. To satisfy this criterion, designers may be forced to increase the geometric parameters of the pile, which increase the material, transportation, and installation costs. Hence, more studies need to be conducted in order to establish the most appropriate combination of parameters that maximize the stiffness of the structure without increasing the overall cost of the turbine.

Improvements in the deterministic models (both analytical and numerical) have been conducted to reduce the foundation cost considering the SLS criteria. Several researchers studied the interaction between soil and pile using finite element (FE) models and quasi-static loads [3], [9]. Posterior researches focused on the effects of the dynamic loading, due to wave and wind excitations, over the foundation response considering parked-conditions, which is the assumption that the rotor behaves as a lumped mass at the hub of the OWT [4], [10]. Zuo et al. [11] on the other hand, developed an FE model that enables the study of the dynamic response of an OWT, while including the effects that the blades exert over the OWT when they are rotating, i.e. non-parking condition. They found that the assumption of parking conditions, widely employed in early research, may result in serious effects on the deflections of the OWT structure. Although improvements in the deterministic models provide valuable information about the shortcomings in the monopile foundations, uncertainties in the model inputs are usually not taken into consideration. As a result, the reliability of the OWT may not be computed properly.

Despite the recognized importance of uncertainty quantification (UQ) in the reliability of the system, few research works have been published which take into consideration the variability of the inputs parameters in OWT foundations [3], [5]. All these studies concluded that the system reached higher deflections employing probabilistic analysis in comparison with deterministic analysis. Moreover, the reliability of the system dropped down when additional sources of uncertainty, were added to the probabilistic analysis. Although these researches attempted to study the effects of uncertainties on the tower deflections, they do not provide a systematic procedure to quantify the uncertainties of the input parameters in an efficient manner other than just assuming probability distributions for these parameters. As a result, there is still a gap in the field of OWT foundations that must be addressed in order to allow design engineers to design cheaper OWT substructures without undermining the system reliability.

In this study, a probabilistic analysis of the dynamic response of a monopile OWT is conducted by using a systematic UQ framework to deal with the uncertainty assessment of the model input parameters. The proposed dynamic model computes the dynamic response of the turbine due to wind and waves loads on the monopile structure utilizing a simple cantilever beam analytical model. The uncertainties of the model input parameters are determined considering the scenarios where: (1) significant amount of data is available using non-parametric statistics, and (2) few data are available by means of the maximum entropy principle (MaxEnt). Monte Carlo simulations are performed to propagate the uncertainties of the model inputs and determine the reliability of the system in terms of the SLS criteria. Comparison of our results with other approaches which assume that the model input parameters are normally distributed shows the validity of the proposed probabilistic framework to ensure the structural reliability of OWT.

\section{METHODOLOGY}

In this work, a probabilistic framework to determine the probability of failure of the structure based on the SLS criterion for a $5[\mathrm{MW}]$ OWT with monopile foundation embedded in clay soil is developed. The framework is carried out in MATLAB and combines a deterministic model to determine the dynamic response of the turbine, and a probabilistic model to quantify and propagate the uncertainties into the deterministic model to obtain the probability density function (PDF) of the model response.

\subsection{Deterministic Model}

The framework used in the deterministic analysis is illustrated in FIGURE 1. The analysis is divided primarily in a load model and a dynamic model. The load model aims to determine the loads exerted on the structure due to the action of the environmental loads, whereas the second one aims to establish the maximum displacement at the hub of the OWT. The surrogate dynamic model, thereafter, is verified and its response is contrasted with the SLS design criterion.

\subsubsection{Wind and Wave Load Models}

The main loads exerted on the structure correspond to environmental loads, aerodynamic and hydrodynamic, which in this work are assumed to be acting in the same direction that in turn represent the most conservative scenario [9], [10]. The wind load exerting on the structure is modeled using the thrust force which can be estimated using Eq. (1) as is suggested in [4], [5], [10]. The turbulent wind speed time series, $U(z, t)$, at rated 
conditions $(12 \mathrm{~m} / \mathrm{s})$ is simulated using the software FAST coupled with TurbSim

$$
F_{U}=0.5 \pi R_{t}^{2} \rho_{a} c_{t} U^{2}(z, t)
$$

The wave loads exerted on the pile are modeled as a point load at the mean sea level using the Morison's equation that is given by

$$
F_{w}=F_{w i}+F_{w d}
$$

where the inertial and drag components of the wave loads, $F_{w i}$ and $F_{w d}$ are

$$
\begin{gathered}
F_{w i}=\int_{-h}^{\eta(z)} 0.25 \rho_{W} c_{m} \pi D^{2} \ddot{W}(z, t) d z \\
F_{w d}=\int_{-h}^{\eta(z)} 0.5 * \rho_{W} * c_{d} * D *|\dot{W}(z, t)| \dot{W}(z, t) d z
\end{gathered}
$$

wherein the wave speed and acceleration, $\dot{W}(z, t) \& \ddot{W}(z, t)$, components were computed assuming the Airy wave theory [5], [6], [9]. A summary of the rest of the parameters employed in this model such as air density, $\rho_{a}$, water density, $\rho_{w}$, thrust coefficient, $c_{t}$, turbine parameters, such as rotor radius, $R_{t}$, and material properties are summarized in Table 1.

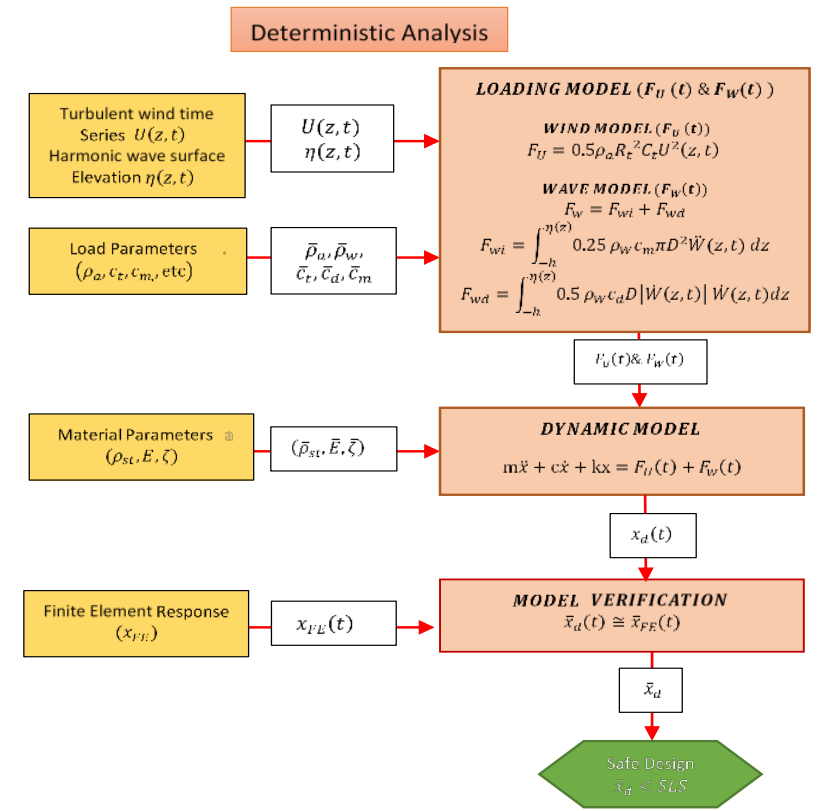

FIGURE 1: DETERMINISTIC FRAMEWORK OF THE OWT MODEL

\subsubsection{Dynamic Model of the Monopile Structure}

The tower-pile structure is modeled as a single degree of freedom oscillator with constant thickness and fixed to the seabed. The model represented in equation (3) is employed to compute the tower displacement at a hub height using time intervals of $0.05 \mathrm{~s}$, and a time span of $30 \mathrm{~s}$. The selection of the analytical model arises from the need to curb the significant computational cost of that an $\mathrm{FE}$ transient analysis requires to obtain the turbine response; however, to ensure the accuracy of the proposed analytical dynamical model, a FE model is carried out in ANSYS to compare and verify the suitability of the proposed analytical model

$$
m \ddot{x}+c \dot{x}+k x=F_{U}(t)+F_{w}(t) .
$$

Table 1: DETERMINISTIC PARAMETERS USED IN THE SIMULATIONS

\begin{tabular}{lc}
\hline Parameter & Value \\
\hline Air density $\left(\boldsymbol{\rho}_{\boldsymbol{a}}\right)$ & $1.23 \mathrm{~kg} / \mathrm{m}^{3}$ \\
Sea water density $\left(\boldsymbol{\rho}_{\boldsymbol{w}}\right)$ & $1,027 \mathrm{~kg} / \mathrm{m}^{3}$ \\
Thrust coefficient $\left(\boldsymbol{c}_{\boldsymbol{t}}\right)$ & 0.75 \\
Drag coefficient $\left(\boldsymbol{c}_{\boldsymbol{d}}\right)$ & 0.7 \\
Mass coefficient $\left(\boldsymbol{c}_{\boldsymbol{m}}\right)$ & 2 \\
Turbulence intensity factor & $16 \%$ \\
Significant wave height & $6 \mathrm{~m}$ \\
Spectral peak frequency & $0.1 \mathrm{~Hz}$ \\
Rotor radius $\left(\boldsymbol{R}_{\boldsymbol{t}}\right)$ & $63 \mathrm{~m}$ \\
Tower and Pile diameter (D) & $6 \mathrm{~m}$ \\
Steel density $\left(\boldsymbol{\rho}_{\boldsymbol{s}}\right)$ & $7850 \mathrm{~kg} / \mathrm{m}^{3}$ \\
Damping ratio $(\boldsymbol{\zeta})$ & $12 \%$ \\
Young modulus $(\mathbf{E})$ & $200 \mathrm{GPa}$ \\
\hline
\end{tabular}

\subsection{Probabilistic Model}

The probabilistic model presented in this work comprises a UQ scheme to systematically assess and propagate the uncertainties of the input random variables (RV) through the deterministic solvers, and a statistical certification measurement to establish the reliability of the structure based on the six RV employed in this analysis. A schematic illustration of the model can be seen in FIGURE 2, in which the light blue boxes represent the UQ of the model inputs and the yellow boxes the uncertainty propagation approach to obtain the PDF of the response.

\subsubsection{Uncertainty Quantification Scheme}

The proposed UQ scheme considered in this study can be seen in FIGURE 3. The first step in the scheme is to classify the type of information associated with the input random variables (RV). Based on that information, the UQ approach in terms of the PDF for each input parameter is selected. The information related to the input random variables can be divided into two main categories, namely, (1) theoretical information (e.g. support, mean, variance) and, (2) experimental-monitored information (significant datasets from experiments or field measurements). As a result, two different approaches are proposed to handle such uncertainties in the least bias fashion. The first approach arises from the information theory and is called MaxEnt. This method aims to establish the PDF of the input RV that maximizes the PDF entropy based on the level of information collected at the moment of the analysis. Mathematically, the MaxEnt is expressed by 


$$
S=-\int f_{X}(x) \ln f_{X}(x) d x
$$

where $f_{X}(x)$ is the joint PDF of the random vector $X$ composed by the input RV, and respect the $M+1$ constraints defined by the known information about $X$

$$
\int g_{k}(x) f_{X}(x) d x=m_{k}
$$

where $g_{k}(x)$ and $m_{k}$ for $k=0,1, \ldots, M$ are known real functions and values, respectively with $g_{0}(x)=1$ and $m_{0}=1$. The maximization problem is solved using the Lagrange multipliers $\lambda_{k}$ for $k=0,1, \ldots, M$ as [12], [13]

$$
f_{X}(x)=\mathbb{I}_{[a, b]}(x) \exp \left(-\lambda_{0}\right) \exp \left[\sum_{k=1}^{N}-\lambda_{k} g_{k}(x)\right]
$$

where $[a, b]$ is the know information about the support of the $\mathrm{RV}$. The second approach uses non-parametric (NP) techniques to establish the uncertainty of the input RV. In the present study, the NP techniques used are the kernel density estimator (KDE) and empirical cumulative distribution function (ECDF). NP approaches produce a realistic representation of the random process that generates the population data only when the sampled data is proved to be statistically significant. The significance of a dataset is tested using the mean-square convergence criterion. This criterion, essentially, look at the convergence of the sample statistics (mean and standard deviation) at a certain number of samples $n_{c}$, which is independent of the size of the dataset [13].

As mentioned before, seven parameters from the load models $\left(\rho_{w}, \rho_{a}, c_{t}, c_{d}, c_{m}, U(x, t)\right)$ and three from the dynamic model $\left(\rho_{s}, \zeta, E\right)$, are considered as independent random variables. Furthermore, two deterministic models are considered for uncertainty propagation (FIGURE 2). The PDFs of air and water densities are determined using MaxEnt with the obtained information for their respective supports and mean values. The PDFs of the thrust, drag, and mass coefficients are also modeled using MaxEnt, but including an extra piece of information related to the support for the coefficient of variation (COV). Additionally, the variability of the wind inflow is modeled following a similar approach, in which the turbulence intensity factor (TIF) is assumed to be proportional to the COV of the wind speed. The Young modulus $(E)$, on the contrary, is the only one estimated using a kernel density approach - that is- because a significant data set of $\mathrm{E}$ for structural steel was available for this analysis[14]. A summary of all RVs considered in the simulations, the available information, and the method used to model their uncertainties can be found in Table 2 .

Once the PDF of the input RVs are determined either by the

MaxEnt or the KDE, random samples are drawn from each

PDFs using the inverse transform method. Those samples are

then used to propagate uncertainties in the input RVs to the response parameters using Monte Carlo simulation (MCS). The last step of the framework is post processing, in which the samples of the response parameters are employed to construct the non-parametric PDFs using KDE and ECDF [13].

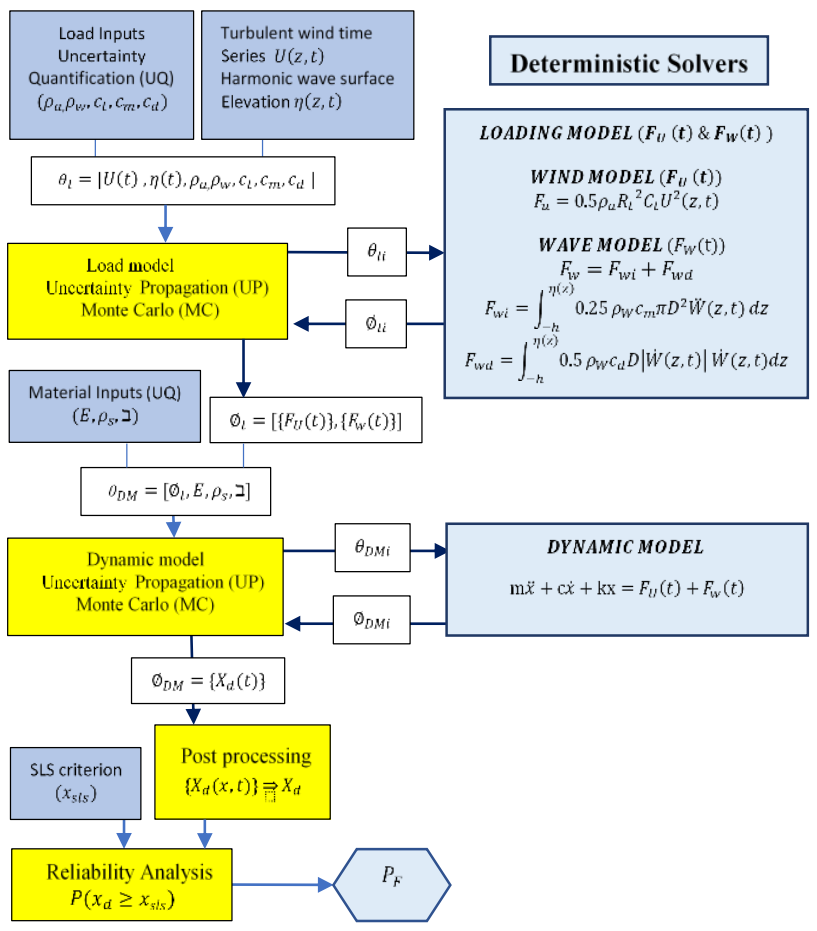

FIGURE 2: PROBABILISTIC FRAMEWORK OF THE OWT MODEL

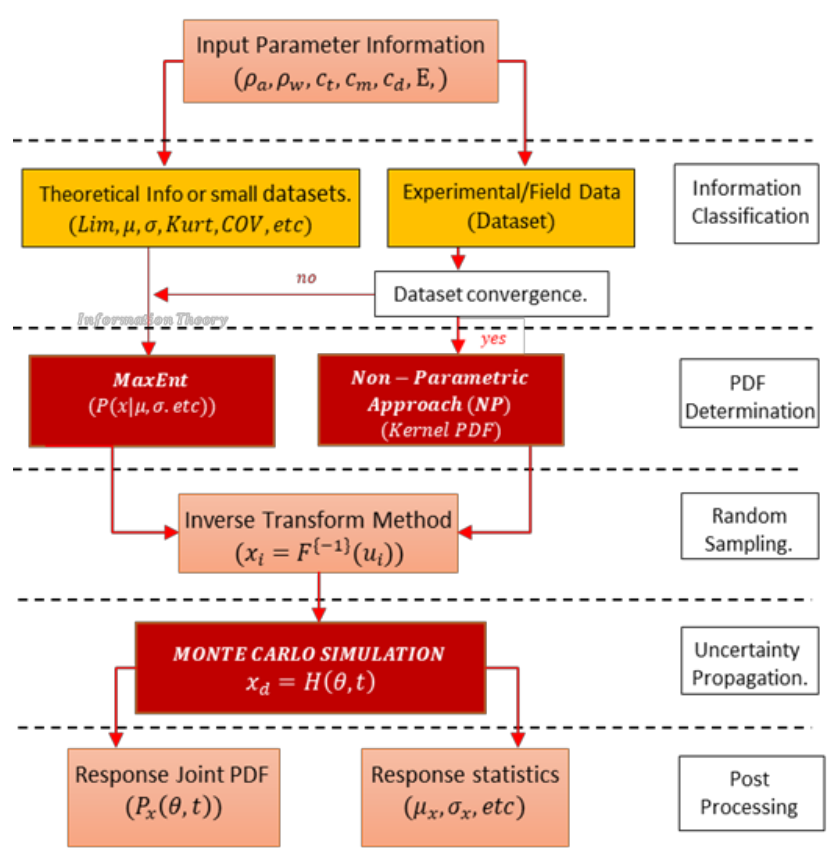

FIGURE 3: UNCERTAINTY QUANTIFICATION SCHEME OF THE RANDOM VARIABLES CONSIDERED IN THIS STUDY 


\subsection{Statistical Certification (Probabilistic Response)}

The statistical certification of the results is represented in terms of a confidence bound plot, and the structural probability of failure associated with the SLS criterion. In this work, a 98\% confidence bound plot is developed based on the sample information at each time step of the displacement stochastic process PDF $\left\{X_{d}(x, t)\right\}$. On the other hand, the probability of failure of the structure, $p_{f}$ (which is also defined as $1-R$, where $R$ is the reliability of the structure), is determined by computing the probability that the limit state function, $Z$, of the response would be equal to zero for a time step $t_{i}[10]$

$$
Z=\operatorname{SLS}-X_{d}\left(x, t_{i}\right)
$$

Table 2: INFORMATION ABOUT THE RANDOM VARIABLES USED IN THE SIMULATIONS

\begin{tabular}{|c|c|c|}
\hline $\mathbf{R V}$ & Information & Method \\
\hline$\rho_{w}$ & $\begin{array}{c}\text { Mean: } 1,027 \mathrm{~kg} / \mathrm{m}^{3} \\
\text { Limits: }[1,020-1,030] \mathrm{kg} / \mathrm{m}^{3}\end{array}$ & MaxEnt \\
\hline$\rho_{a}$ & $\begin{array}{c}\text { Mean: } 1.23 \mathrm{~kg} / \mathrm{m}^{3} \\
\text { Limits: }[1.146-1.315] \mathrm{kg} / \mathrm{m}^{3}\end{array}$ & MaxEnt \\
\hline$c_{t}$ & $\begin{array}{c}\text { Mean: } 0.75 \\
\text { Limits: }[0-1] \\
\text { COV: }[0.2-10] \%\end{array}$ & MaxEnt \\
\hline$c_{d}$ & $\begin{array}{c}\text { Mean: } 0.7 \\
\text { Limits: }[0-1] \\
\text { COV: }[0.2-10] \%\end{array}$ & MaxEnt \\
\hline$c_{m}$ & $\begin{array}{c}\text { Mean: } 0.2 \\
\text { Limits: }[0-1] \\
\text { COV }=[0.2-10] \%\end{array}$ & MaxEnt \\
\hline$U\left(x, t_{i}\right)$ & $\begin{array}{cl} & \text { Mean: } U\left(t_{i}\right) \\
\text { Limits: } & {\left[U\left(t_{i}\right)+/-0.4 * U\left(t_{i}\right)\right]} \\
& \text { TIF }=[10-22] \%\end{array}$ & MaxEnt \\
\hline$\rho_{s}$ & $\begin{array}{c}\text { Mean: } 7850 \mathrm{~kg} / \mathrm{m}^{3} \\
\text { Limits: }[5495-10205] \mathrm{kg} / \mathrm{m}^{3} \\
\text { COV }=[0.5-15] \%\end{array}$ & MaxEnt \\
\hline$\zeta$ & $\begin{array}{c}\text { Mean: } 0.16 \\
\text { Limits: }[0.72-1.68] \\
\text { COV }=[0.5-15] \%\end{array}$ & MaxEnt \\
\hline $\mathbf{E}$ & Dataset of 41 [samples] & $\begin{array}{c}\text { Non- } \\
\text { Parametric }\end{array}$ \\
\hline
\end{tabular}

\section{RESULTS AND DISCUSSION}

In this work a probabilistic framework considering an analytical dynamic model to compute the total displacement at hub height applied for an OWT is proposed.

\subsection{Deterministic Results}

The verification of the analytical model using an FE model can be seen in FIGURE 4. From the graph, it is apparent that after the transient effects from 0 to 8 seconds approximately, the FE model and the analytical model responses have virtually the same mean and relatively follows the same trend. Hence, the proposed analytical may be employed as a suitable approximation for computing the turbine response instead of computationally expensive FE simulations, wherein several simulations are required.

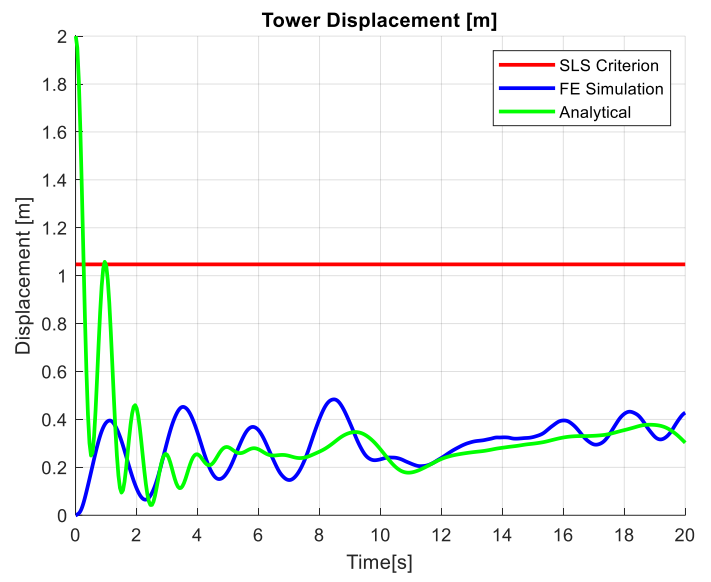

FIGURE 4: DETERMINISTIC SOLUTION OF THE TOWER DISPLACEMENT AT THE HUB HIGHT USING THE FINITE ELEMENT MODEL AND THE ANALYTICAL MODEL

\subsection{UQ Results}

FIGURE 5 shows the PDFs (and the random samples drew out of them) of the input RVs determined using either the MaxEnt or the KDE approaches. For the air density and seawater density PDFs shown in FIGURE 5(a) and (b) the MaxEnt with information about their support and mean values are used; as a result, truncated exponential distributions with the curve rise close to the mean value are obtained. In FIGURE 5(c), (d), (e), (f) and (g), the parameters $c_{t}, c_{d}, c_{m}, \rho_{s}$ and $\xi$, are determined using the MaxEnt with information regarding their mean, limits and a variable coefficient of variation (COV) ranging from 2 to $15 \%$. From the graphs, it is clearly seen that the shape of the MaxEnt PDF determined out this information shows a defined bell curve, wherein the peak of the distribution coincides the model parameter mean value. Despite the apparent similarities with either a Gaussian distribution or any other standard distribution, the MaxEnt PDFs are calculated considering the maximum level of uncertainty based on the available information. Consequently, the MaxEnt, in contrast with assumed distributions, ensures the minimum level of bias on the analysis.

On the other hand, FIGURE 5(h) shows the PDF obtained using the KDE for a statistically significant sample of the material Young modulus. FIGURE 6 shows the convergence plots of three random arrangements of the same dataset in order to find the number of samples wherein convergence is achieved, which is approximately 35 samples for the analyzed dataset. It is important to mention that although NP methods are the best estimators when data is available, the failure to establish the convergence of the dataset would produce a bias non-parametric approximation of the true distribution. Therefore, when meansquared convergence is not achieved, NP methods may result in inaccurate probability distributions for the hub height displacement. 

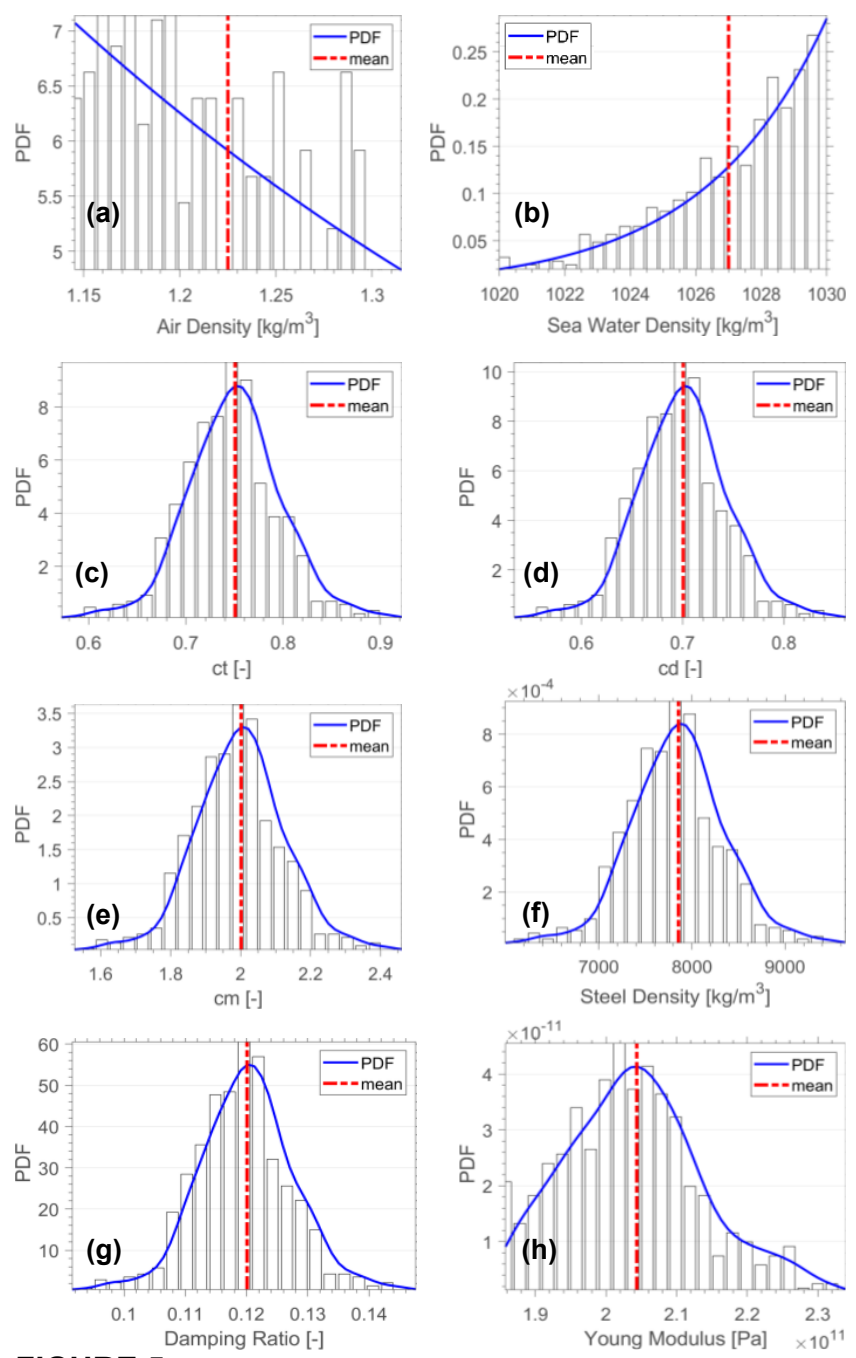

FIGURE 5: PROBABILITY DENSITIES OF THE MODEL INPUT RANDOM VARIABLES: (a) AIR DENSITY, (b) SEA WATER DENSITY, (c) THRUST COEFFICIENT, (d) DRAG COEFFICIENT, (e) MASS COEFFICIENT, (f) YOUNG MODULUS, (g) STEEL DENSITY, AND (h) DAMPING RATIO
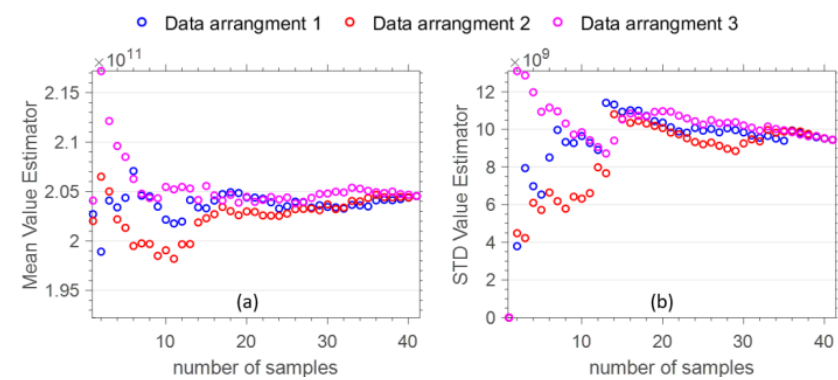

FIGURE 6: CONVERGENCE OF THE ESTIMATORS: (a) MEAN VALUE AND (b) STANDARD DEVIATION) OF THE YOUNG MODULUS DATASET USING THE MEAN-SQUARE CRITERION

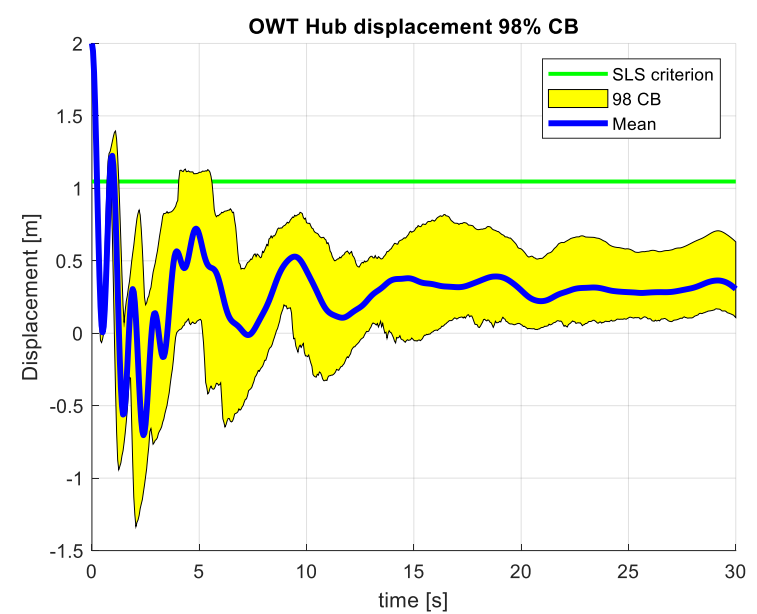

FIGURE 7: PROBABILISTIC RESULTS FOR THE OWT HUB DISPLACEMENT WITH 98\% CONFIDENCE BOUND

\subsection{Probabilistic Results}

FIGURE 7 shows the $98 \%$ confidence bound of the mean displacement response of the turbine at the hub height. The confidence bound indicates that $98 \%$ of the points around the sample mean (blue line) are included in the band. Such limits may vary if (1) more sources of uncertainty are added in the analysis, i.e. wave load, or (2) more accurate information, i.e. experiments, regarding the parameters included in the analysis are included. Both cases are considered as future research.

\subsubsection{Effects of Simplified UQ Schemes in the Probabilistic Response}

In an attempt to quantitative illustrate the shortcoming of the utilization of a simplified UQ scheme, the proposed framework is contrasted with a simulation that is carried out assuming normality for the RVs considering in this study. FIGURE 8, for instance, shows the differences between the PDFs and samples when the model parameter distributions are determined using the MaxEnt (FIGURE 8a) and arbitrarily assumed as a normal distribution (FIGURE 8b). From the graph, it is clearly seen the scattering of the samples (from 0.8 to 1.7 approximately) generated from the normal distribution is considerably larger than the ones out of the MaxEnt distribution counterpart (roughly 1.15 to 1.3 ). Such differences may add bias and extra variability to the analysis because the samples generated outside of the stated air density limits, i.e.1.15 to 1.3 , are rarely or highly unlikely to be observed in reality.

In addition, the selection of the approach has significant importance in the determination of the probability of failure based on the SLS criterion. In order to show the influence of applying other approaches, a reliability analysis is conducted at $t_{i}=4[s]$. The results of the prior analysis, such as the entire cumulative distribution function (CDF) of the $Z$ function $(Z)$, and a zoom of the left tail of the CDF that highlight the PF of the OWT structure can be seen in FIGURE 9(a) and (b), 
respectively. From FIGURE 9(a) it is apparent that the CDF computed based on a normal distribution (NCDF) differs significantly from the ECDF computed from the samples obtained from MC simulations in the tails, in which the value of the $P_{f}$ (or reliability) is established. Such offset can be illustrated in detail in FIGURE 9(b), in which it is clearly seen that the $P_{f}$ calculated utilizing the NCDF $(5.5 \%)$ is considerably lower than the $P_{f}$ obtained from the ECDF $(8.8 \%)$. As a result, the arbitrary assumption of distributions can result in an underestimation of the structural probability of failure, which in turn may mean an underestimation of the reliability of the structure.
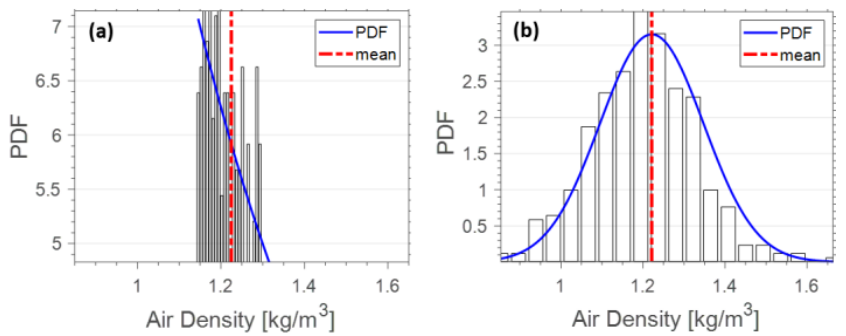

FIGURE 8: PROBABILITY DENSITIES OF THE AIR DENSITY USING: (a) MAXENT AND, (b) NORMAL DISTRIBUTION
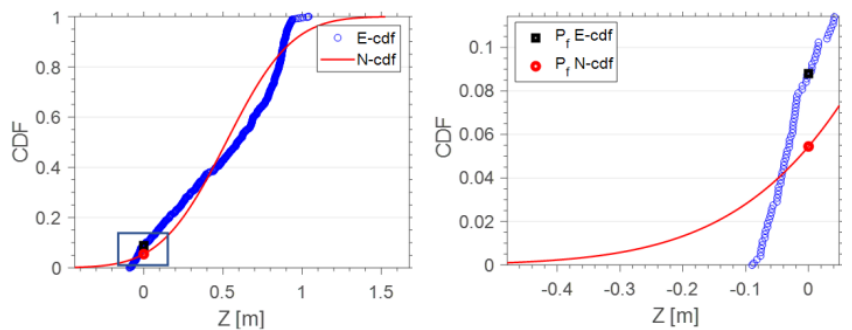

FIGURE 9: CDF COMPARISON OF THE LIMIT STATE FUNCTION OF THE DISPLACEMENT AT 5[s]: (a) ENTIRE CDF AND, (b) ZOOM OF THE CDF LEFT TAIL.

\section{CONCLUSION}

In conclusion, the inherent variability of the parameters that comprise the different models to determine the OWT dynamic response can yield the highest values than their deterministic counterpart. Additionally, the arbitrary selection of PDF can produce misleading estimations of the reliability of the system. Therefore, further research will have (1) a more realistic deterministic model, which will include the tower shadowing effects, a stochastic wave model and soil model; (2) a more accurate stochastic model that accounts for more sources of uncertainties; (3) and other failure modes, i.e. fatigue. All the aforementioned strategies aim to improve the proposed UQ framework in order to obtain more robust reliability estimations.

\section{REFERENCES}

[1] V. Negro, M. D. Esteban, and C. Matutano, "Uncertainties in the design of support structures and foundations for offshore wind turbines," Renew. Energy, vol. 63, pp. 125-132, 2014.

[2] V. J. Kurian, S. P. Narayanan, and C. Ganapathy, "Towers for offshore wind turbines," in Proceedings of the 10th Asian International Conference on Fluid Machinery, 2010, pp. 1-10.

[3] W. Carswell, S. R. Arwade, D. J. DeGroot, and M. A. Lackner, "Soil-structure reliability of offshore wind turbine monopile foundations," Wind Energy, vol. 18, pp. 483-498, 2015.

[4] S. Bisoi and S. Haldar, "Dynamic analysis of offshore wind turbine in clay considering soil-monopile-tower interaction," Soil Dyn. Earthq. Eng., vol. 63, no. August, pp. 19-35, 2014.

[5] S. Haldar, J. Sharma, and D. Basu, "Probabilistic analysis of monopile-supported offshore wind turbine in clay," Soil Dyn. Earthq. Eng., vol. 105, no. November 2017, pp. 171-183, 2018.

[6] S. Bhattacharya, "Challenges in Design of Foundations for Offshore Wind Turbines," Eng. Technol. Ref., pp. 19, 2014.

[7] Y. E. Mostafa and M. H. El Naggar, "Response of fixed offshore platforms to wave and current loading including soil-structure interaction," Soil Dyn. Earthq. Eng., vol. 24, no. 4, pp. 357-368, 2004.

[8] B. Zhu, K. Wen, D. Kong, Z. Zhu, and L. Wang, "A numerical study on the lateral loading behaviour of offshore tetrapod piled jacket foundations in clay," Appl. Ocean Res., vol. 75, no. September 2017, pp. 165-177, 2018.

[9] R. Rezaei, P. Fromme, and P. Duffour, "Fatigue life sensitivity of monopile-supported offshore wind turbines to damping," Renew. Energy, vol. 123, pp. 450459, 2018.

[10] S. Bisoi and S. Haldar, "Design of monopile supported offshore wind turbine in clay considering dynamic soilstructure-interaction," Soil Dyn. Earthq. Eng., vol. 73, pp. 103-117, 2015.

[11] H. Zuo, K. Bi, and H. Hao, "Dynamic analyses of operating offshore wind turbines including soil-structure interaction," Eng. Struct., vol. 157, no. November 2017, pp. 42-62, 2018.

[12] A. Cunha Jr, "Modeling and Quantification of Physical Systems Uncertainties in a Probabilistic Framework," Probabilistic Progn. Heal. Manag. Energy Syst., pp. 127-156, 2017.

[13] J. P. Dias, S. Ekwaro-Osire, A. Cunha Jr., S. Dabetwar, A. Nispel, F. M. Alemayehu, and H. B. Endeshaw, "Parametric probabilistic approach for cumulative fatigue damage using double linear damage rule considering limited data", Int. J. Fatigue, vol. 127, no. October 2019, pp. 246-258, 2019.

[14] A. Haldar and S. Mahadevan, Probability, Reliability, and Statistical Methods in Engineering Design, 1st ed. John Wiley \& Sons, Inc., 2000. 